\title{
Dysphagia in Elderly Women: Consider Tetanus
}

\author{
A. Rauch, S. Droz, S. Zimmerli, S. L. Leib
}

\begin{abstract}
Background: Dysphagia is seldom caused by tetanus; however, it is a common symptom of tetanus. Treating patients with tetanus is a rare event in industrialized countries and awareness is needed to recognize early signs of this serious disease. In Switzerland, the most recently reported tetanus cases occurred in elderly women with insufficient seroprotection.

Patients: We report on three elderly women presenting with dysphagia as an initial symptom of tetanus.

Results: Generalized tetanus was diagnosed in two patients upon admission, the third presented with cephalic tetanus with secondary generalization. All three patients had undetectable levels of tetanus antibodies and had no documented prior tetanus immunizations. Cultures of wound swabs grew Clostridium tetani in all cases. Electromyography was highly suggestive for tetanus in two patients. Treatment involved mechanical ventilation, intravenous benzodiazepine and metronidazole therapy, and active and passive tetanus immunization. The disease had a favorable outcome in two cases and was fatal in one.
\end{abstract}

Conclusion: Tetanus remains a threat in patients with insufficient seroprotection and efforts are needed to improve tetanus immunization in these individuals. Tetanus should be considered in the differential diagnosis of dysphagia.

Infection 2006; 34: 35-38

DOI 10.1007/s15010-006-4151-7

\section{Introduction}

Tetanus has become a rare disease in industrialized countries. In Switzerland, the annual incidence of tetanus decreased from 4.3 to 0.4 cases/million population between 1979 and 2004 [1]. Zuber et al. [2] estimated that only about $10 \%$ of tetanus cases are reported to the Swiss Federal Office of Health, but tetanus would be a rare event even if $100 \%$ of cases were reported. Hullstrung et al. [1] found antibodies to tetanus in $36(92 \%)$ of 39 elderly patients in a Swiss geriatric clinic, and interestingly, the median concentration of anti-tetanus antibodies in males was more than twice as high as in females. This gender-specific difference in antitetanus serum titer is likely the result of repeated booster immunizations of adult male Swiss citizens during their annual weeks of compulsory military service. The fact that over $75 \%$ of tetanus cases observed in Switzerland over the last 5 years occurred in women older than 60 years further supports the concept that gender-specific differences in anti-tetanus serum titer leads to an increased incidence of tetanus in this risk group. Surveillance data for 1998-2000 in the United States showed that adults older than 60 years were at highest risk for tetanus (annual incidence of 0.35 cases/million population) and tetanus-related death (casefatality ratio of $40 \%$ ) [3]. Dysphagia is a common symptom of tetanus [4]. However, because dysphagia is seldom caused by tetanus, the correct diagnosis and adequate therapy are likely to be delayed. The purpose of this report is to outline the presentation and clinical course of three patients with dysphagia as a common symptom of tetanus.

\section{Patients \\ Case 1}

A 75-year-old woman was admitted to the emergency department with dysphagia and slight neck stiffness. Ten days before admission, the patient was in her usual good health when she fell with her left knee into a rusty iron rod which penetrated the skin and underlying structures to a depth of approximately $1 \mathrm{~cm}$. The wound became red, hot and purulent 3 days after this incident. One day before admission she reported dysphagia and had increasing difficulties opening her mouth. The general practitioner performed wound debridement, active immunization for tetanus, and organized immediate hospitalization. Medical history was significant for bilateral knee prosthesis 15 years ago. The patient lived in a rural area in Switzerland and had not traveled recently. She had no known prior tetanus immunizations.

Physical examination revealed a slightly increased tone in the masseter muscles and a tender $3 \times 4 \mathrm{~cm}$ wound with purulent discharge on the left knee (Figure 1). The patient was alert
S. Droz, S. Zimmerli, S.L. Leib (corresponding author)

Institute for Infectious Diseases, University of Bern, Friedbühlstrasse 51, P.O. Box 61, 3010 Bern, Switzerland; Phone: (+41/31) 632-4949, Fax: -3550, e-mail: stephen.leib@ifik.unibe.ch

A. Rauch, S. Zimmerli, S.L. Leib

Clinic and Policlinic for Infectious Diseases, University Hospital,

Inselspital, Bern, Switzerland 


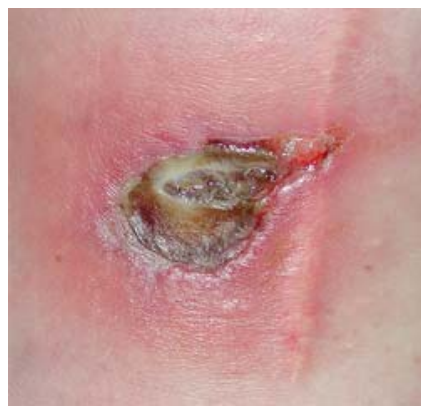

Figure 1. Case 1: Macroscopic aspect of the wound over the left knee at admission. The surrounding skin was erythematous and hot. Culture of the purulent wound secretion revealed growth of Clostridium tetani and Clostridium perfringens.

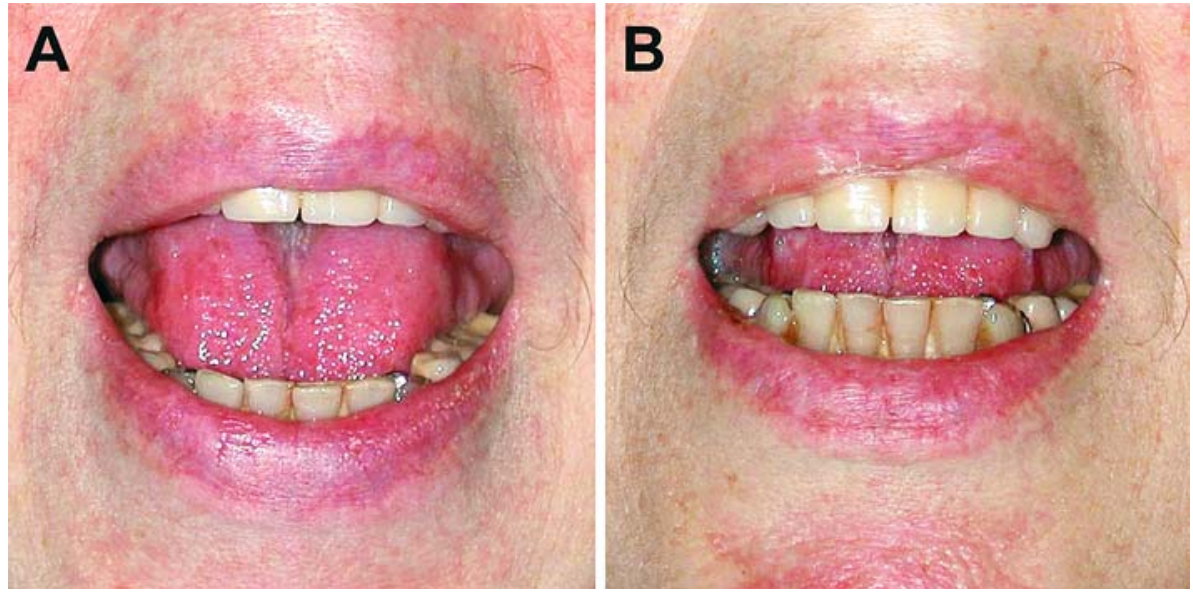

Figure 2. Case 1: Evolution of symptomatic trismus. The patient was instructed to maximally open her mouth at admission (A) and on the following day (B). and coherent, and all other physical and neurological findings were normal. Laboratory studies showed no abnormalities. Tetanus antibodies were not detectable by the enzyme-linked immunosorbent assay (ELISA). Electromyography of the masseter muscles showed spontaneous activity of motor units which could not be suppressed voluntarily and an absence of the silent period. Laboratory exams and clinical evaluation revealed no signs of strychnine intoxication, of hypocalcemia or of a dystonic reaction. The diagnosis of tetanus was made and therapy started including human tetanus immunoglobulin (4,000 IU i.m.), metronidazole (500 mg i.v. qid) and lorazepam (i.v. in 2-mg increments). Swabs taken from the bottom of the wound during debridment were cultured aerobically and anaerobically. All aerobic cultures revealed Staphylococcus aureus. After 48 h of incubation, the anaerobic cultures revealed two different morphologies. The two gram-positive rods were identified as Clostridium tetani and Clostridium perfringens. C. tetani isolates are routinely identified to the species level by volatile fatty acid analysis, and by sequencing of 1,507-bp of the 16S rRNA gene.

Within 1 day, the patient developed rapidly progressive trismus (Figure 2), painful muscle spasms, respiratory insufficiency, and autonomic dysfunction necessitating intubation under sedation and relaxation. She needed to be mechanically ventilated for 4 weeks. Repeated wound debridement resulted in complete healing of the soft-tissue infection. The patient was released from the intensive care setting after 6 weeks. She recovered almost completely; the only sequelae were slight dysphagia and an impaired gait.

\section{Case 2}

An 86-year-old woman sustained a head injury while gardening. Wound débridement was performed immediately. Although her tetanus immunization history was unknown, she received at that time neither tetanus toxoid nor tetanus immunoglobulin. One week later, the patient was hospitalized with progressive dysphagia and trismus and received active tetanus immunization, human tetanus immunoglobulin (500 IU i.m.) and metronidazole (500 $\mathrm{mg}$ i.v. tid) upon admission. Within 2 days of hospital admission, she developed respiratory failure and required mechanical ventilation. Clinical findings and electromyography were highly suggestive of generalized tetanus. The swabs taken from the facial wound were cultured aerobically and anaerobically. The aerobic cultures showed no growth after $48 \mathrm{~h}$ of incubation but anaerobic cultures revealed three different morphologies. The three gram-positive rods were identified as $C$. tetani, $C$. sporogenes and $C$. hastidiforme. Tetanus antibodies were not detectable by ELISA. The patient developed severe autonomic dysfunction and progressive opisthotonus. Both spasms and autonomic dysfunction progressed rapidly despite adequate intensive care management. The patient died 6 days after admission.

\section{Case 3}

An 85-year-old woman presented to the emergency department with an (initially unrecognized) orbital floor fracture and a facial wound from a wooden stick. Wound débridement and active tetanus immunization were performed immediately, but no tetanus immunoglobulins were given at that time. She had no known prior tetanus immunizations. The patient was readmitted to the hospital 2 weeks later with an infraorbital wound infection and diplopia due to an orbital floor fracture, which required surgical reconstruction. Five days later, she developed dysphagia, facial paresis, trismus and subsequently progressive respiratory insufficiency. The aerobic cultures taken from the orbita abscess during operative revision revealed Serratia liquefaciens and mixed gram-positive bacteria which were not further investigated. No anaerobic bacteria were observed on the anaerobe solid media. Owing to the high clinical suspicion of tetanus, the thioglycolate medium was subcultured and grew multiple anaerobes. The grampositive rod was identified as $C$. tetani. The other anaerobes were not further specified. Tetanus antibodies were not detectable by ELISA. Electromyographic findings were not conclusive. A diagnosis of cephalic tetanus with secondary generalization was considered and therapy was complemented with human tetanus immunoglobulin (1,000 IU i.m.), metronidazole (500 mg i.v. tid) and intravenous midazolam (in 2.5-mg increments). Initially, the muscle spasms involving head, neck, and the arms intensified despite sedative and anticonvulsant therapy. After 4 weeks, the spasms improved and the patient was extubated. She recovered well and was discharged 2 months after admission to a rehabilitation center.

\section{Discussion}

The three cases described above illustrate important epidemiological, clinical, diagnostic and therapeutic aspects of tetanus in industrialized countries. We discuss here these issues with respect to our cases. 
All three cases presented in this report were elderly women, had no documented prior tetanus immunizations and had undetectable levels of tetanus antibodies. This finding reflects the particular risk for tetanus in elderly women outlined in the introduction and underscores the importance of providing adequate tetanus immunization to the whole population.

Tetanus diagnosis is primarily based on clinical symptoms [5, 6]. Dysphagia as an initial symptom of tetanus has been described in case reports, primarily from otolaryngologic departments [7-13]. In a 1957 review of 91 patients with tetanus, dysphagia was noted in $10 \%$ at admission to the hospital and developed subsequently in $50 \%$ of patients [4]. On admission to an Australian hospital, trismus was present in $93 \%$ and dysphagia in $46 \%$ of 106 patients with tetanus [14]. In an otolaryngologic center in Turkey, $92 \%$ of 37 patients with tetanus had trismus and $78 \%$ dysphagia as initial symptoms of the disease [15]. In a Belgian hospital, all 27 patients with tetanus developed dysphagia and trismus during hospitalization [16]. Dysphagia was the first symptom reported by our patients; a detailed clinical history, however, revealed additional symptoms of tetanus in all three cases which underscores the importance of clinical awareness for tetanus in this setting. Wound cultures grew C. tetani in all cases presented in this report. However, the diagnostic value of positive cultures is limited: A positive culture may be present without disease and does not indicate whether the organism contains the toxin-producing plasmid. Electromyography has shown to be helpful in the diagnosis of tetanus, but as illustrated in Case 3, inconclusive results do not rule out tetanus [17]. The patient presented in Case 3 suffered most probably from cephalic tetanus with secondary generalization. This special form of initially localized disease affects primarily the cranial nerves and commonly follows an injury to the head. It is characterized by lower motoneuron lesion, frequently producing facial paresis [18]. Paralysis is maximal close to the site of injury, while spasm is evident at more distant sites [19]. Like in other forms of localized tetanus, patients with cephalic tetanus often proceed to develop generalized tetanus [18, 19].

Standard treatment of tetanus includes benzodiazepines, opiates and vasoactive drugs to help reduce muscle spasms, pain, and autonomic dysfunction [20,21]. Benzodiazepines remain the mainstay of sedative and anticonvulsant treatment [22]. Some reports suggest an additional benefit of intrathecal baclofen [23]. Passive immunization is given to neutralize circulating toxin. Data on the effect of different doses of tetanus immune globulin suggest that $500 \mathrm{U}$ may be as effective as higher doses of 3,000-10,000 U [24]. Prior tetanus does not provide immunity, possibly due to the minimal amounts of toxin needed to cause disease. Thus, passive immunization and active immunization with tetanus toxoid should commence early in the management of the patient $[20,21]$. The choice of antibiotic therapy remains controversial. A study comparing oral metronidazole to intramuscular penicillin showed improved survival in the metronidazole group, possibly due to the GABA antagonist effects of penicillin $[25,26]$.

The introduction of intensive care treatment has substantially reduced mortality, but mortality rate is still high, particularly in elderly persons [27]. The patient with fatal outcome reported in Case 2 presented several features which have been associated with an unfavorable outcome: a short incubation period, an internal or facial site of infection and lack of immune protection [28].

Tetanus remains a threat in persons with insufficient seroprotection. Treating patients with tetanus is a rare event in industrialized countries and awareness is needed to recognize early signs of this rare but serious disease. Tetanus should be considered in the differential diagnosis of dysphagia.

\section{Acknowledgments}

Funding was received from the Swiss National Science Foundation, grant No. 632-66057.01.

\section{References}

1. Hullstrung HD, Mausezahl D, Feuz $M$, Herzog C, Conzelmann $M$, Zimmerli W: Tetanus immunisation in geriatric patients with accidental wounds: how much is needed? Swiss Med Wkly 2003; 133: 227-232.

2. Zuber PL, Schierz A, Arestegui G, Steffen R: Tetanus in Switzerland 1980-1989. Eur J Epidemiol 1993; 9: 617-624.

3. Pascual FB, McGinley EL, Zanardi LR, Cortese MM, Murphy TV: Tetanus surveillance - United States, 1998-2000. MMWR Surveill Summ 2003; 52:1-8.

4. Christensen NA, Thurber DL: Clinical experience with tetanus: 91 cases. Mayo Clin Proc 1957; 32: 146-158.

5. Farrar JJ, Yen LM, Cook T, Fairweather N, Binh N, Parry J, Parry CM: Tetanus. J Neurol Neurosurg Psychiatry 2000; 69: 292-301.

6. Henderson SO, Mody T, Groth DE, Moore JJ, Newton E: The presentation of tetanus in an emergency department. J Emerg Med 1998; 16: 705-708.

7. Stephenson JW, Blacklay B: A case of tetanus presenting as dysphagia. J Laryngol Otol 1955; 69: 496-498.

8. Weider DJ, Tingwald FR: Dysphagia as initial and prime symptom of tetanus. Report of a case. Arch Otolaryngol 1970; 91: 479-481.

9. Wang L, Karmody CS: Dysphagia as the presenting symptom of tetanus. Arch Otolaryngol 1985; 111: 342-343.

10. Kasanzew M, Browne B, Dawes P: Tetanus presenting as dysphagia. J Laryngol Otol 1989; 103: 229-230.

11. Scholz DG, Olson JM, Thurber DL, Larson DE: Tetanus: an uncommon cause of dysphagia. Mayo Clin Proc 1989; 64: 335-338.

12. Ross J, Murrant NJ: Dysphagia as a major symptom of tetanus. J Laryngol Otol 1992; 106: 923-924.

13. Mastaglia FL, Thickbroom GW, Day T, Bond R: Craniocervical tetanus presenting with dysphagia: diagnostic value of electrophysiological studies. J Neurol 2001;248:903-904

14. Newton-John HF: Tetanus in Victoria, 1957-1980. Review of 106 patients managed in one hospital. Med J Aust 1984; 140: 194-200.

15. Aydin K, Caylan R, Bektas D, Koksal I: Otolaryngologic aspects of tetanus. Eur Arch Otorhinolaryngol 2003; 260: 52-56. 
16. Peetermans WE, Schepens D: Tetanus - still a topic of present interest: a report of 27 cases from a Belgian referral hospital. J Intern Med 1996; 239: 249-252.

17. Steinegger T, Wiederkehr M, Ludin HP, Roth F: Electromyography as a diagnostic aid in tetanus. Schweiz Med Wochenschr 1996; 126: $379-385$.

18. Jagoda A, Riggio S, Burguieres T: Cephalic tetanus: a case report and review of the literature. Am J Emerg Med 1988; 6: 128-130.

19. Dastur FD, Shahani MT, Dastoor DH, Kohiyar FN, Bharucha EP, Mondkar VP, Kashyap GH, Nair KG: Cephalic tetanus: demonstration of a dual lesion. J Neurol Neurosurg Psychiatry 1977; 40: 782-786.

20. Hsu SS, Groleau G: Tetanus in the emergency department: a current review. J Emerg Med 2001; 20:357-365.

21. Cook TM, Protheroe RT, Handel JM: Tetanus: a review of the literature. Br J Anaesth 2001; 87: 477-487.

22. Okoromah CN, Lesi FE: Diazepam for treating tetanus. Cochrane Database Syst Rev 2004:CDo03954.
23. Santos ML, Mota-Miranda A, Alves-Pereira A, Gomes A, Correia J, Marcal N: Intrathecal baclofen for the treatment of tetanus. Clin Infect Dis 2004; 38: 321-328.

24. Blake PA, Feldman RA, Buchanan TM, Brooks GF, Bennett JV: Serologic therapy of tetanus in the United States, 1965-1971. JAMA 1976; 235: 42-44.

25. Ahmadsyah I, Salim A: Treatment of tetanus: an open study to compare the efficacy of procaine penicillin and metronidazole. Br Med J (Clin Res Ed) 1985; 291: 648-650.

26. Weiss DS, Hablitz JJ: Interaction of penicillin and pentobarbital with inhibitory synaptic mechanisms in neocortex. Cell Mol Neurobiol 1984; 4: 301-317.

27. Anonymous: Leads from the MMWR. Tetanus - United States, 1982-1984. JAMA 1985; 254:2873, 2877-2878.

28. Phillips LA: A classification of tetanus. Lancet 1967; 1: 1216-1217. 